\title{
NOUVELLE
}

\section{La pêche au gène chez le poisson zèbre Identification du transporteur du fer mitochondrial}

Sophie Vaulont, Lydie Viatte

\author{
Inserm U567, CNRS UMR-S 8104, \\ Faculté de Médecine René Descartes, Institut Cochin, \\ 24, rue du Faubourg Saint-Jacques, 75014 Paris, France. \\ vaulont@cochin.inserm.fr \\ viatte@cochin.inserm.fr
}

> La pêche a souvent été miraculeuse en utilisant le poisson zèbre comme système génétique pour cloner de nouveaux gènes impliqués dans l'hématopoïèse et le métabolisme du fer (pour revue, voir [1]). Quelle en est la méthode? Le principe est simple. Après mutagenèse chimique, les embryons de poisson ayant des signes d'anémie sont sélectionnés. Cette opération est facile car les œufs de poisson, qui sont fécondés dans le milieu extérieur, sont optiquement clairs, permettant ainsi l'observation directe de la circulation des cellules sanguines. Des groupes de complémentation sont ensuite définis et la caractérisation du gène muté responsable du défaut phénotypique est réalisée par clonage positionnel. Ainsi, plus de 26 groupes de complémentation ont été caractérisés et déjà une dizaine de gènes, impliqués pour la plupart dans des pathologies du fer chez l'homme, ont été clonés (Tableau I) ${ }^{1}$. C'est cette stratégie de clonage positionnel qui vient d'être utilisée par une équipe américaine pour identifier le gène responsable de la mutation frascati, frs, chez le poisson zèbre [2].

Les embryons mutants frs présentent, $36 \mathrm{~h}$ après fécondation, un compartiment érythropoïétique très largement diminué : ces embryons développent une anémie sévère par arrêt de la différenciation des érythrocytes au stade pro-érythroblaste. Le nombre d'embryons mutants qui se développent jus-

${ }^{1}$ Que les amateurs de vin se tiennent sur leurs gardes, ces mutants ne sont pas recommandés pour la dégustation... qu'à l'état adulte est très faible. Les rares survivants sont pâles, présentent un retard de croissance et une forte cardiomégalie, signes classiques d'insuffisance cardiaque. Le gène responsable de l'anomalie phénotypique du mutant frs code pour une protéine apparentée à la famille des transporteurs mitochondriaux SLC25 [3] et baptisée mitoferrine (mfrn) par les auteurs. L'ADNc pleine longueur de la mitoferrine

a été cloné chez le poisson zèbre; il code une protéine de 333 acides aminés d'une masse calculée de $37 \mathrm{kDa}$. Le gène mitoferrine s'exprime de façon abondante et spécifique dans la masse cellulaire intermédiaire de l'embryon de poisson (l'équivalent du sac vitellin extra-embryonnaire de mammifères). Cette expression embryonnaire est sous la dépendance du facteur de transcription Gata-1, facteur essentiel pour la

\begin{tabular}{|c|c|c|}
\hline Mutant & Protéine & Maladie chez l'homme \\
\hline chardonnay & $\begin{array}{l}\text { Divalent Metal Transporterl } \\
\text { (DMTl) }\end{array}$ & Anémie microcytaire \\
\hline chablis & Protéine 4.lr & Elliptocytose héréditaire \\
\hline chianti & Récepteur de la transferrine 1 & - \\
\hline dracula & Ferrochélatase & Protoporphyrie érythroïde \\
\hline moonshine & TIFl $\gamma$ & - \\
\hline retsina & Band3 & $\begin{array}{l}\text { Anémie dysérythropoïétique } \\
\text { congénitale }\end{array}$ \\
\hline riesling & Spectrine $\beta$ & Sphérocytose héréditaire \\
\hline sauternes & Aminolévulinate synthase $\delta$ & Anémie sidéroblastique congénitale \\
\hline vlad tepes & Gata-1 & $\begin{array}{l}\text { Anémie dysérythropoḯtique } \\
\text { familiale et thrombocytopénie }\end{array}$ \\
\hline weissherbst & Ferroportine & Hémochromatose de type 4 \\
\hline yquem & Uroporphyrinogène décarboxylase & $\begin{array}{l}\text { Porphyrie cutanée tardive } \\
\text { et porphyrie hépato-érythroïde }\end{array}$ \\
\hline zinfandel & Locus globine & Thalassémie \\
\hline frascati & Mitoferrine & $?$ \\
\hline
\end{tabular}

Tableau I. Gènes clonés chez le poisson zèbre et leurs implications dans des maladies chez l'homme. 
régulation de l'érythropoïèse terminale. Dans le poisson zèbre adulte, on retrouve les transcrits mitoferrine dans le rein, site de l'hématopoïèse. Enfin, la distribution subcellulaire de la mitoferrine a été déterminée après transfection d'une protéine chimérique mitoferrine-GFP dans des cellules épithéliales humaines. Comme toutes les protéines de la famille SLC25, la mitoferrine est retrouvée dans la membrane interne des mitochondries. Pour valider l'effet perte de fonction de la mutation mitoferrine, des expériences classiques de sauvetage du phénotype (rescue) ont été réalisées par micro-injection d'ARNc sauvage chez les embryons mutants. Dans ces conditions, $50 \%$ des embryons se développent normalement et présentent une hémoglobinisation identique à celle des embryons sauvages. À l'inverse, des expériences d'invalidation fonctionnelle de la protéine chez l'embryon sauvage ${ }^{2}$

${ }^{2}$ Ces expériences sont réalisées par micro-injection chez l'embryon de morpholinos (oligomères stables modifiés chimiquement qui se lient à l'ARN) qui ont pour but de bloquer spécifiquement l'épissage du pré-messager mitoferrine et donc la production de la protéine.

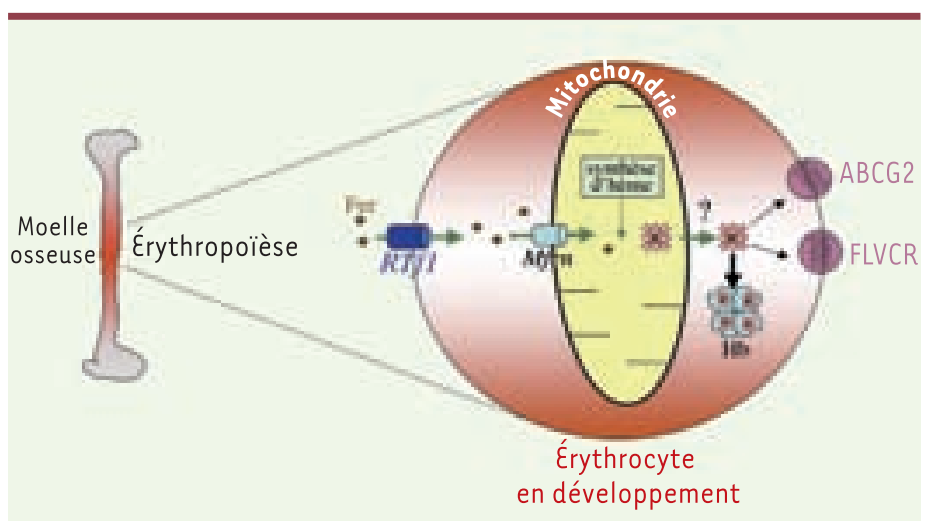

Figure 1. Modèle de la fonction de la mitoferrine dans la mitochondrie. Le fer est capté par l'érythrocyte en développement grâce au récepteur de la transferrinel (RTfl). Il est ensuite transporté dans la mitochondrie par la mitoferrinel (Mfrn). C'est dans la mitochondrie que se déroule une grande partie de la synthèse de l'hème, en particulier la dernière étape catalysée par la ferrochélatase qui assure la fixation du fer sur la protoporphyrine IX. L'hème quitte alors la mitochondrie (soit passivement à travers la membrane, soit par un processus actif non encore identifié) pour se lier à la chaîne de globine en croissance et former I'hémoglobine $(\mathrm{Hb})$. L'hème peut également sortir de la cellule grâce à deux exporteurs récemment caractérisés, FLVCR et ABCG2, permettant ainsi d'éviter les excès de fer qui entraîneraient l'apoptose des précurseurs érythroïdes (pour revue, voir[5]). montrent des résultats phénotypiques très similaires à ceux observés chez les embryons frs, à savoir une anémie hypochrome avec arrêt de la différenciation érythroïde.

Chez la souris, l'expression du gène mitoferrine est exclusivement restreinte aux tissus hématopoïétiques, à savoir le foie fœtal, la rate et la moelle osseuse. Un deuxième gène a été identifié, mitoferrine2, chez le poisson zèbre et les mammifères. Notons que l'expression du gène mitoferrine2 de souris est ubiquiste et que I'ARNc mitoferrine2 n'est pas capable de sauver le phénotype des embryons frs, soulignant l'absence de redondance fonctionnelle entre mitoferrinel et 2 pour l'érythropoïèse.

Pour établir le rôle exact de la mitoferrine, et donc le lien entre la perte de fonction de la protéine et l'acquisition du phénotype d'anémie du mutant frs, les auteurs ont établi une lignée de cellules \&S (embryonic stem cells) déficientes en mitoferrinel (cellules $\mathrm{mfrn}^{-/-}$). Les cellules souches $E S$ peuvent être induites vers un lignage hématopoïétique sui-

vant un processus bien établi de culture et en présence d'un cocktail spécifique d'agents différenciateurs. Lorsque ces cellules ES « hématopoiétiques » sont cultivées enprésence d'érythropoiétine et d'interleu$\mathrm{k}$ in e- 3 , une différenciation érythroïde spécifique peut être obtenue. Dans ces conditions, aucune induction érythroïde n'est observée avec les cellules ES hématopoïétiques $\mathrm{mfrn}^{-/-}$, contre $15 \%$ de cellules érythroïdes différenciées obtenues à partir de cellules ES $\mathrm{mfrn}^{+/+}$et $7 \%$ à partir de cellules $\varepsilon S \mathrm{mfrn}^{+/-}$. Cette absence de différenciation érythroïde pouvant venir d'un défaut d'incorporation du fer dans la mitochondrie (compartiment où se déroule en partie la synthèse de l'hème) (Figure l), les auteurs ont testé l'incorporation de fer radioactif $\left({ }^{55} \mathrm{Fe}\right)$ dans l'hème mitochondrial. Pour cela, les cellules $\varepsilon S$ hématopoïétiques ont été incubées en présence de transferrine saturée en ${ }^{55} \mathrm{Fe}$ (internalisée dans la cellule grâce au récepteur de la transferrinel présent à la surface des cellules) et d'acide aminolévulinique, le précurseur de l'hème. Alors que les cellules ES hématopoïétiques sauvages incorporent le ${ }^{55} \mathrm{Fe}$ de façon efficace, aucune incorporation de ${ }^{55} \mathrm{Fe}$ n'est obtenue dans la molécule d'hème avec les cellules $\varepsilon S \mathrm{mfrn}{ }^{-/-}$. Ces résultats indiquent clairement que la mitoferrine est indispensable pour l'hémoglobinisation des cellules en permettant l'incorporation de ${ }^{55} \mathrm{Fe}$ au sein de la molécule d'hème.

De façon intéressante, les protéines mitoferrine de poisson partagent $38 \%$ d'identité avec les protéines MRS3 et MRS4 de levure impliquées dans le transport mitochondrial de métaux [4]. Le double mutant mrs3/4 de levure présente un défaut de biogenèse des protéines $\mathrm{Fe}-\mathrm{S}$ et des hémoprotéines qui se traduit par un ralentissement de la croissance. Ce défaut de croissance est «sauvé » par la mitoferrinel ou 2 de poisson et la mitoferrine2 humaine. Ces résultats soulignent la très grande conservation de la fonction de la mitoferrine à travers les espèces (la mitoferrine de souris «sauve » le mutant frs de poisson et la mitoferrine de poisson complémente le mutant de levure mrs3/4). Cependant, comme précédemment mentionné, seule la mitoferrinel est capable de «sauver » l'embryon mutant frs. Ce résultat peut 
s'expliquer par un besoin minimal en fer de la levure qui peut être assuré par la mitoferrine2 (de même, dans les cellules non-érythroïdes du poisson, le transfert mitochondrial du fer est probablement assuré par la mitoferrine2). En revanche, les quantités importantes de fer nécessaires au cours de la différenciation érythroïde semblent pouvoir n'être suppléées que par la mitoferrinel.

Enfin, la dernière série d'expériences démontrant l'activité de la mitoferrine dans l'import de fer mitochondrial a utilisé des clones stables de levure mrs3/4 exprimant un gène rapporteur codant pour une enzyme mitochondriale dont l'activité est dépendante du fer. Les auteurs montrent que l'activité de cette enzyme est réduite de moitié dans la souche mutante mrs3/4. Après transfection de I'ADNc de la mitoferrine de poisson, cette activité retourne à la normale, indiquant bien que la mitoferrine a permis au fer de rentrer dans la mitochondrie et, par la même, d'activer l'enzyme.

En conclusion, ce travail a permis de caractériser la protéine responsable du transport du fer mitochondrial, la mitoferrinel pour les précurseurs érythroïdes et, probablement, la mitoferrine2 pour les cellules non-érythroïdes. Ce transport de fer est crucial pour la production mitochondriale de l'hème, composant essentiel du métabolisme du fer. En effet, on retrouve l'hème non seulement dans l'hémoglobine du globule rouge mais également dans la myoglobine, la neuroglobine ainsi que toutes les enzymes à groupement prosthétique (catalase, peroxydase, cytochrome, nitric oxid synthase...). II y a fort à parier que tout dérèglement de la mitoferrine soit responsable de pathologie(s) chez l'homme. $\diamond$ Gene fishing in zebrafish :

identification

of the iron mitochondrial transporter

\section{RÉFÉRENCES}

1. De Jong JL, Zon LI. Use of the zebrafish system to study primitive and definitive hematopoiesis. Annu Rev Genet $2005 ; 39$ : 481-501.

2. Shaw GC, Cope JJ, Li L, et al. Mitoferrin is essential for erythroid iron assimilation. Nature2006 ; 440: 96-100.

3. Wohlrab H. The human mitochondrial transport protein family: identification and protein regions significant for transport function and substrate specificity. Biochim Biophys Acta2005; 1709 : 157-68.

4. Li L, Kaplan J. A mitochondrial-vacuolar signaling pathway in yeast that affects iron and copper metabolism. J Biol Chem 2004; 279 : 33653-61.

5. Latunde-Dada G0, Simpson RJ, McKie AT. Recent advances in mammalian haem transport. Trends Biochem Sci 2006 ; 31 : 182-8.

\section{NOUVELLE}

La duplication du gène APP,
cause de maladie d'Alzheimer
associée à une importante
angiopathie amyloide

Dominique Campion, Didier Hannequin

> Lorsqu'en 1906 Alois Alzheimer décrivit la maladie qui porte son nom, il rapporta la présence de lésions particulières dans le cerveau des patients: les plaques séniles extracellulaires et les dégénérescences neurofibrillaires intracellulaires. Il fallut attendre le milieu des années 1980 et les travaux respectifs de G. Glenner et J.P. Brion pour que les constituants majeurs de ces deux lésions soient caractérisés: il s'agit, d'une part, d'un peptide de 39 à 42 acides aminés, le peptide $A \beta$, produit lors du clivage séquentiel d'une protéine intramembranaire nommée APP (amyloid precursor protein) et, d'autre part, d'une protéine liée aux microtubules, la protéine Tau. Les analyses génétiques menées depuis 15 ans ont montré que le déterminisme de la maladie d'Alzheimer est complexe. Dans la majorité des cas, il est polyfactoriel. Un facteur de risque génétique impliqué dans ces formes communes, l'allèle $\varepsilon 4$ du gène de l'apolipoprotéine $\varepsilon$, a été identifié. Dans une minorité de cas, le déterminisme est autosomique dominant avec pénétrance complète à l'âge de 60 ans. Des mutations de type faux sens sur deux gènes, le gène $A P P$ et le gène de la préséniline 1 ( $P S E N 1$ ), sont responsables de la grande majorité de ces formes mendéliennes à début pré-
Inserm U614,

Faculté de Médecine-Pharmacie de Rouen, 22, boulevard Gambetta,

76183 Rouen Cedex 01, France.

dominique.campion@univ-rouen.fr

coce. Les études menées au cours des années 1990 ont montré que la conséquence de ces diverses altérations génétiques était univoque. Dans tous les cas, elles s'accompagnent d'une surproduction du peptide $A \beta$ 42, qui est la forme la plus agrégable de ce peptide. Les mutations identifiées sur le gène APP sont essentiellement localisées au niveau des sites de clivage du peptide $A \beta$ sur son précurseur et interfèrent avec ce clivage. La préséniline 1 est, pour sa part, un membre essentiel du complexe $\gamma$-sécrétase, 